\title{
EFICÁCIA DE Beauveria bassiana PARA O CONTROLE DE Hedypathes betulinus EM ERVA-MATE, Ilex paraguariensis
}

\author{
Larissa Rolim Borges ${ }^{1}$, Sônia Maria Noemberg Lazzari ${ }^{2}$, \\ Lívia Rolim Borges-Arriagada ${ }^{3}$, Edson Tadeu Iede ${ }^{4}$ \\ ${ }^{1}$ Bióloga, Dr ${ }^{\mathrm{a}}$., Instituto Agronômico de Pernambuco, Recife, PE - larissarolimborges@gmail.com \\ ${ }^{2}$ Bióloga, Dr ${ }^{\text {. }}$, Depto. de Zoologia, UFPR, Curitiba, PR, Bolsista do CNPq - lazzari@ufpr.br \\ ${ }^{3}$ Eng$^{\mathrm{a}}$ Florestal, Especialista, Meco Madeiras Remanufaturadas Ltda., Fazenda Rio Grande, PR - lívia-borges@uol.com.br \\ ${ }^{4}$ Biólogo, Dr., EMBRAPA Florestas, CNPF, Colombo, PR - iedeet@cnpf.embrapa.br \\ Recebido para publicação: 04/11/2009 - Aceito para publicação: 18/08/2010
}

\begin{abstract}
Resumo
O controle das pragas da erva-mate, dentre as quais a broca-da-erva-mate, Hedypathes betulinus, é limitado principalmente a técnicas silviculturais e catação manual, pois não é permitida a aplicação de agroquímicos nessa cultura. A fim de contribuir com informações para o controle biológico de $H$. betulinus, o presente trabalho teve por objetivos determinar a eficácia e o número de aplicações anuais do micoinseticida à base do fungo entomopatogênico Beauveria bassiana para o controle dessa praga. Os experimentos foram conduzidos em uma área de erva-mate em monocultura, no município de Campo Alegre, SC, Brasil. O formulado foi pulverizado no tronco e na base das árvores, de acordo com um cronograma de duas ou três aplicações, em diferentes meses do ano. Constatou-se a eficácia com a utilização do formulado, sendo que os dados de captura das áreas tratadas diferiram estatisticamente da testemunha e do tratamento com catação manual. Contudo, não houve diferença estatística entre os tratamentos com duas e três aplicações, sendo capturados, respectivamente, $18,4 \%$ e $17,7 \%$ dos insetos marcados. Assim, apenas duas pulverizações com B. bassiana realizadas nos meses de novembro e fevereiro são eficientes para o controle de $H$. betulinus e manutenção do inóculo na área de erva-mate em monocultura.

Palavras chave: Fungo entomopatogênico; controle biológico; flutuação populacional.
\end{abstract}

\begin{abstract}
Beauveria bassiana efficiency to Hedypathes betulinus control in yerba maté, Ilex paraguariensis. Yerba mate pest control, especially Hedypathes betulinus, is limited basically to silvicultural practices and insect removal, once application of chemical insecticides in such crop is not allowed. This research aimed to produce data for biological control of $H$. betulinus by evaluation of efficiency and number of applications of an entomopathogenic fungus Beauveria bassiana formulation for control of such pest species. The experiment was carried out on a yerba maté monoculture area, in Campo Alegre county, Santa Catarina, Brazil. The fungus was sprayed on trees' trunk, following a chronogram of two and three applications, in different months. Efficiency of B. bassiana formulation could be demonstrated by the number of recaptured insects in areas of application, which was statistically lower than the one in the control area. However, there was no significant difference between two and three fungus application, with, respectively $18.4 \%$ and $17.7 \%$ of recaptured insects. Thus, only two yearly B. bassiana applications, in November and February, are enough to control $H$. betulinus and keep inoculum in an area of yerba maté monoculture.

Keywords: Entomopathogenic fungi; biological control; insect fluctuation.
\end{abstract}

\section{INTRODUÇÃO}

A erva-mate, Ilex paraguariensis St.-Hil. (Aquifoliaceae), é uma cultura importante e tradicional no sul do Brasil, e em função do seu crescente consumo os produtores têm implantado povoamentos puros onde antes havia florestas nativas (PENTEADO et al., 2000). Assim, quando cultivada a pleno sol, essa espécie ombrófila fica sujeita a estresse fisiológico que a torna mais suscetível ao ataque de pragas e doenças (CARPANEZZI, 1995; DIAZ, 1997; IEDE, 1985; LEITE et al., 2006a; SOARES, 1998). Em

FLORESTA, Curitiba, PR, v. 41, n. 2, p. 313-320, abr./jun. 2011. 
função disso, diversas espécies de insetos associados a ervais nativos tiveram um grande aumento populacional, tornando-se pragas e provocando sérios danos à cultura, dentre as quais a broca-da-ervamate, Hedypathes betulinus (KLUG, 1825) (Coleoptera: Cerambycidae) (DIAZ, 1997; PENTEADO, 1995; SOARES, 1998).

A adoção de medidas de controle deve estar inserida em um programa de manejo integrado de pragas (MIP), em que diversos métodos de controle podem ser empregados de maneira integrada. A aplicação de agroquímicos apresenta sérias restrições, pois não existem produtos registrados para a cultura. Atualmente, as táticas empregadas para redução de níveis populacionais da praga são a poda, a capina e o coroamento, deixando faixas de vegetação secundária entre as linhas de plantio (LEITE et al., 2006b). Esses métodos eliminam as regiões preferenciais de abrigo das pragas, expondo-as à insolação (dessecação) e ação de predadores, além de favorecer a visualização de $H$. betulinus para a catação manual. Essas práticas também melhoram as condições microclimáticas e a eficácia do controle biológico com fungos entomopatogênicos (SOARES; IEDE, 1997; LEITE et al., 2006b).

$\mathrm{O}$ cultivo da erveira em condições semelhantes às do ambiente natural reduz o estresse da planta, tornando-a mais resistente ao ataque de pragas (SOARES; IEDE, 1997), e o adensamento com espécies nativas favorece a nidificação de pássaros e incrementa as populações de inimigos naturais. Borges et al. (2003) compararam os sistemas de cultivo nativo e adensado e concluíram que uma opção adequada é o adensamento planejado a partir das áreas de erval nativo. Nesse caso, retira-se apenas a vegetação de pequeno porte e espécies sem valor econômico, e plantam-se mudas de procedência garantida, tornando o cultivo mais rentável e mais equilibrado do que o erval nativo e com menor incidência de pragas do que a monocultura.

O método mecânico de supressão populacional de $H$. betulinus mais utilizado pelos produtores é a coleta manual dos adultos, pela sua eficiência e fácil execução (LEITE et al., 2006b). Entretanto requer mão-de-obra intensiva, e quando deixa de ser realizado ocasiona um incremento populacional da brocada-erva-mate, com prejuízos acentuados à cultura (SOARES; IEDE, 1997).

O controle biológico tem sido utilizado para a regulação das densidades populacionais dos insetos considerados pragas em diversas culturas comerciais. Entre os agentes mais utilizados estão os parasitoides, predadores e os patógenos (fungos, vírus, bactérias e protozoários) (ALVES, 1998; GALLO et al., 2002). Segundo Bellows (2001), Headrick; Goeden (2001), Lanza et al. (2004) e outros autores, o emprego de fungos entomopatogênicos apresenta-se como uma excelente medida para o controle biológico de diversos insetos, pelo fato de apresentarem baixo impacto ambiental e complementarem outros métodos de supressão populacional (ALVES, 1998). A constatação da ocorrência natural do fungo Beauveria bassiana (Bals.) Vuill. (Deuteromycota) infectando insetos e ácaros da cultura da erva-mate (DALLA SANTA et al., 2009; OLIVEIRA et al., 2002; RIBEIRO, 1994), incluindo adultos de $H$. betulinus (SOARES et al., 1995; SOARES; IEDE, 1997) abre perspectivas para sua utilização, visando assegurar a melhoria fitossanitária e consequentemente a qualidade e produtividade da erva-mate, atendendo às atuais exigências do mercado (BORGES et al., 2003).

Com base nessas considerações, o propósito desta pesquisa foi avaliar a eficácia e determinar o número e época de aplicações de formulado fúngico à base de $B$. bassiana, para o controle de $H$. betulinus em um sistema de monocultivo de erva-mate.

\section{MATERIAL E MÉTODOS}

Os estudos de campo foram conduzidos em um plantio em monocultura de erva-mate, estabelecido em 1998, situado no município de Campo Alegre, SC (26¹1' S, 49¹4' W), ocupando uma área total de aproximadamente 28 ha de erva-mate plantada com espaçamento de 1,5 m x 3,0 m.

O erval experimental foi dividido em quatro áreas de $1 / 2$ ha, cada uma correspondendo a um tratamento: T I - sem qualquer tratamento contra $H$. betulinus (testemunha); T II - catação manual com remoção dos insetos adultos; T III - duas aplicações anuais com o formulado fúngico de $B$. bassiana nos meses de novembro e fevereiro; T IV - três aplicações anuais com formulado fúngico de B. bassiana nos meses de novembro, janeiro e fevereiro. O cronograma de aplicação de B. bassiana a campo foi fundamentado no ciclo biológico do inseto, de acordo com Soares (1998), visando a potencialização do patógeno para o controle de $H$. betulinus. Assim, realizou-se uma aplicação no início da emergência dos adultos (novembro) e a segunda e terceira no período de pico populacional da praga (janeiro e fevereiro). 
Neste estudo, o micoinseticida à base de B. bassiana foi formulado em óleo emulsionável a 0,5\% e aplicado na concentração de 2,7 a $3,5 \times 10^{7}$ conídios $/ \mathrm{mL}$. Essa cepa foi isolada a partir de adultos de $H$. betulinus coletados em campo (LEITE et al., 2000) e encontra-se catalogada na coleção da Embrapa Recursos Genéticos (CENARGEN) sob o código CG 716 (em fase de registro pela Embrapa Florestas e Turfal Ind. e Com. de Produtos Biológicos e Agronômicos).

O formulado foi aplicado no tronco e na base das erveiras com um pulverizador costal de $10 \mathrm{~L}$, equipado com bico hidráulico e ponta de jato cônico vazio durante 15 segundos, utilizando-se aproximadamente $150 \mathrm{~mL}$ de calda/planta. Essa metodologia teve como objetivo não deixar resíduo do fungo nas folhas, que são consumidas "in natura", e restringir a aplicação ao local onde os adultos de $H$. betulinus caminham e ovipositam, evitando a infecção de insetos associados às outras partes da erveira, principalmente os inimigos naturais. O horário de aplicação foi o vespertino (entre 17 e 18 h), período em que é possível evitar os efeitos deletérios da luz ultravioleta e a temperatura, mais amena, é menos prejudicial ao patógeno.

O experimento foi realizado de novembro/2004 a abril/2007, sendo que no período de alta densidade populacional do inseto (novembro a abril) as avaliações foram quinzenais, enquanto que nos períodos de baixa densidade (maio a outubro) foram mensais. Foi feita a amostragem absoluta dos adultos, realizando-se a inspeção visual em todas as 1.000 árvores que compunham cada tratamento. Durante as inspeções, todos os insetos adultos encontrados nos tratamentos T I (testemunha), T III e T IV (com aplicação de B. bassiana) foram retirados da planta, marcados no pronoto com esmalte de cores diferentes (uma cor para cada tratamento) e devolvidos para a área. No tratamento T II (catação) não houve marcação dos insetos, apenas a sua contagem e remoção do erval. Os dados numéricos das coletas foram transformados em "Log x" na base 10 e comparados pelo teste de Duncan (5\%).

\section{RESULTADOS E DISCUSSÃO}

No período entre novembro/2004 e abril/2007 foram realizadas 45 coletas de H. betulinus no erval, sendo perdida uma das coletas de janeiro/2005 e a coleta de agosto/2005. As médias mensais da temperatura $\left({ }^{\circ} \mathrm{C}\right)$, umidade relativa $(\mathrm{UR} \%)$ e precipitação $(\mathrm{mm})$ estão representadas na figura 1.

Em função de grande perda econômica de safras anteriores, o proprietário do erval realizou a retirada por catação dos adultos de $H$. betulinus ao redor das áreas experimentais, no período de dezembro/2004 a março/2005, totalizando cerca de 10.100 insetos, e no período de dezembro/2005 e março/2006, totalizando 4.050 insetos. Em função desse procedimento, houve redução no número de adultos também na área experimental.

$\mathrm{O}$ total de adultos de $H$. betulinus registrados durante os 30 meses da pesquisa, nas quatro áreas experimentais, foi 8.574, sendo $3.369(39,1 \%)$ na área da testemunha sem qualquer tratamento (T I), $2.111(24,6 \%)$ na área de catação manual (T II), $1.581(18,4 \%)$ na área com duas aplicações de $B$. bassiana (T III) e 1.513 (17,7\%) na área com três aplicações de B. bassiana (T IV) (Figuras 2 e 3). Foi observada diferença significativa, a $1 \%$ de probabilidade, entre os tratamentos com fungo (T III e T IV) e a testemunha (T I) e com a catação manual (T II), porém não houve diferença entre os tratamentos com duas e três aplicações (Tabela 1). Esses resultados indicam que os tratamentos com aplicação do formulado fúngico de $B$. bassiana reduzem significativamente as populações de $H$. betulinus em campo. Além disso, observou-se que em 2007, ano em que não foram realizadas novas aplicações fúngicas, não houve redução muito expressiva no número de indivíduos quanto nos anos anteriores. A persistência do inóculo do fungo no campo, até 120 dias após a aplicação, deve-se principalmente à sua disseminação durante a cópula e à presença das carcaças dos insetos mortos, conforme constatado por Borges (2007). Dessa maneira, recomenda-se a aplicação do fungo anualmente, com pelo menos duas aplicações, seguindo o cronograma proposto, para que se mantenha a população do inseto sob controle.

Os dados obtidos neste experimento corroboram resultados de Leite et al. (2000), que testaram, em laboratório, sete linhagens de B. bassiana, uma de Beauveria brongniartii (Sacc.) Petch e uma de Metarhizium anisopliae (Metsch.) Sorokin, obtidas da Embrapa Recursos Genéticos. A linhagem de $B$. bassiana $\mathrm{CG} 716$, na concentração $10^{7}$ conídios $/ \mathrm{mL}$ apresentou os melhores resultados, com mortalidade de $100 \%$ em campo. Entretanto, de acordo com os autores, esse resultado pode ser atribuído ao fato de o fungo ter sido aplicado em toda a erveira ( $300 \mathrm{ml} /$ /erveira), e não apenas no tronco, não sendo considerado o melhor método, pois pode ocasionar a infecção de outros insetos, incluindo os inimigos naturais.

FLORESTA, Curitiba, PR, v. 41, n. 2, p. 313-320, abr./jun. 2011. 


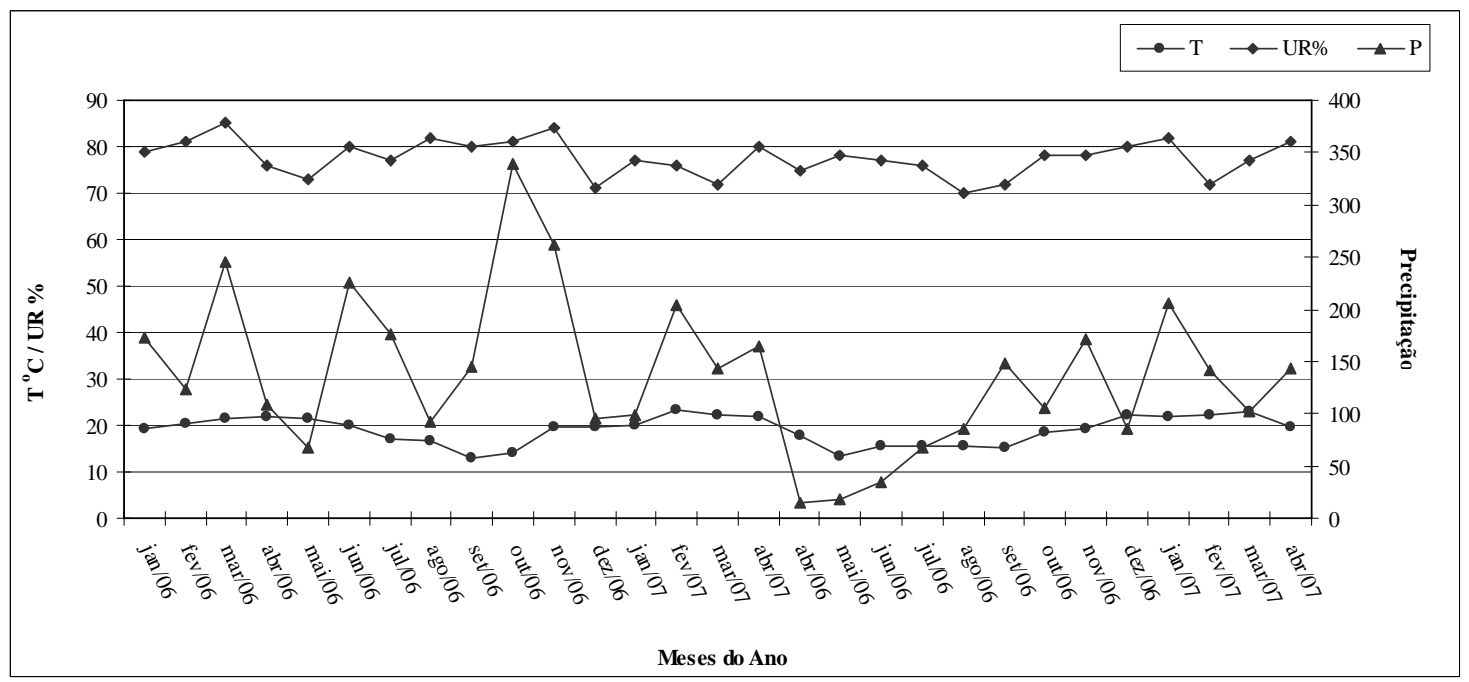

Figura 1. Médias mensais da temperatura $\left({ }^{\circ} \mathrm{C}\right)$, umidade relativa (UR\%) e precipitação $(\mathrm{mm})$ no município de Campo Alegre, SC.

Figure 1. Monthly temperature $\left({ }^{\circ} \mathrm{C}\right)$, relative humidity $(\mathrm{RH} \%)$ and rainfall $(\mathrm{mm})$ in the county of Campo Alegre, SC.

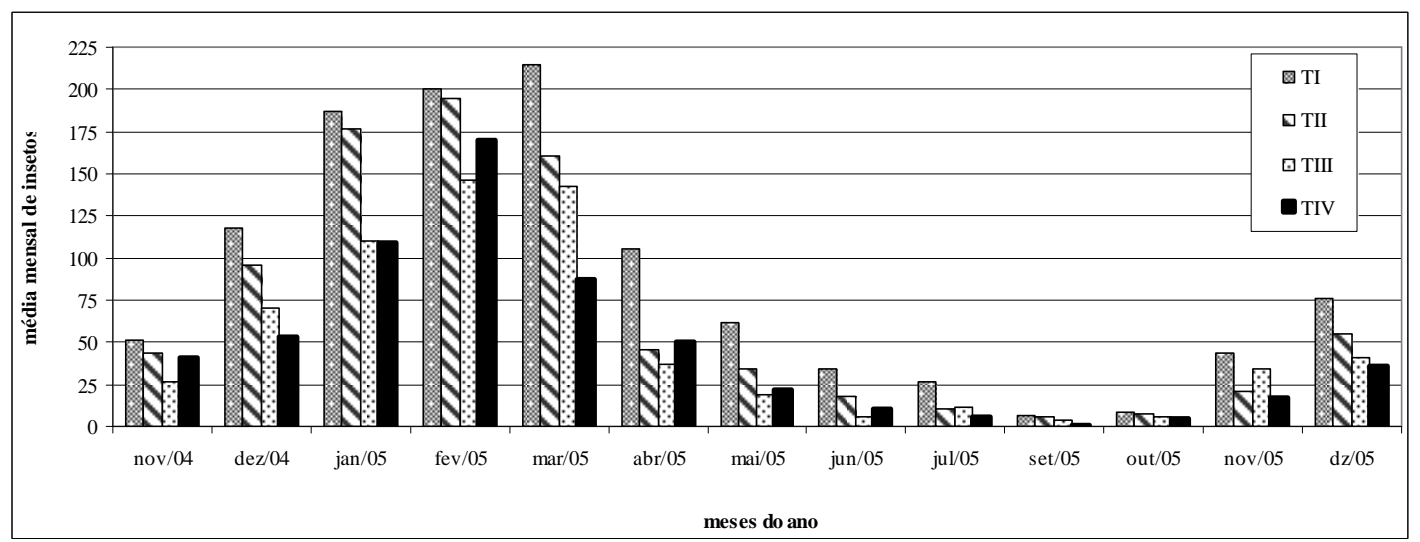

Figura 2. Ocorrência de adultos de H. betulinus nos tratamentos T I (testemunha), T II (catação), T III (duas aplicações anuais de $B$. bassiana), T IV (três aplicações anuais de B. bassiana), de novembro/2004 a dezembro/2005, em um povoamento puro de erva-mate, I. paraguariensis, no município de Campo Alegre, SC.

Figure 2. Occurrence of $H$. betulinus adults in four areas of intensive cultivation of yerba mate, $I$. paraguariensis, under the following treatments: T I (control), T II (hand picking), T III (two applications per year of $B$. bassiana), T IV (three applications per year of B. bassiana), November/04 to December/05 in the county of Campo Alegre, SC.

Outros estudos corroboram a eficiência de $B$. bassiana no controle de $H$. betulinus na concentração usada nesta pesquisa. Testes realizados em laboratório com dois gêneros de fungos entomopatogênicos, B. bassiana (linhagem CG 716) e Paecilomyces sp., na concentração de $10^{7}$ esporos/mL, demonstraram que $B$. bassiana foi mais infectiva, apresentando uma mortalidade de 97,5\%, com tempo médio de 16,8 dias, contra 37,5\% e tempo médio de 32 dias de Paecilomyces sp. (Leite et al., 2003a). Em testes de campo, também com a linhagem CG 716 de B. bassiana na concentração de $10^{7}$ esporos $/ \mathrm{mL}$ aplicada somente no tronco da erveira, obtiveram-se $70,8 \%$ de infectividade com tempo letal médio de 30,6 dias (LEITE et al., 2003b). O poder residual foi avaliado 
durante quatro dias e resultou em infectividade acima de 56\%. A linhagem de B. bassiana (CG 716) também foi testada em campo, formulada em duas concentrações de óleo emulsionado e três concentrações de conídios, sendo que o formulado mais eficiente foi o de concentração $10^{7}$ conídios $/ \mathrm{mL}$ e $0,5 \%$ de óleo, com infectividade de $76 \%$, considerado economicamente viável para produção em larga escala (LEITE et al., 2006a).

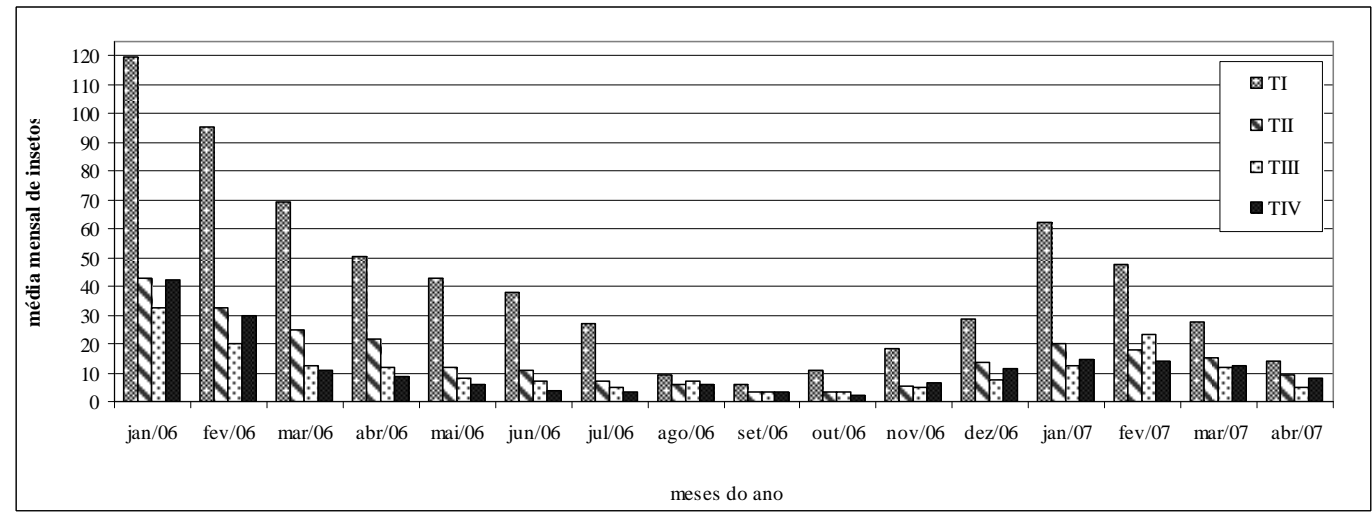

Figura 3. Ocorrência de adultos de H. betulinus nos tratamentos T I (testemunha), T II (catação), T III (duas aplicações anuais de $B$. bassiana), T IV (três aplicações anuais de $B$. bassiana), de janeiro/2006 a abril/2007, em um povoamento puro de erva-mate, I. paraguariensis, no município de Campo Alegre, SC.

Figure 3. Occurrence of $H$. betulinus adults in four areas of intensive cultivation of yerba mate, $I$. paraguariensis, under the following treatments: T I (control), T II (hand picking), T III (two applications per year of B. bassiana), T IV (three applications per year of B. bassiana), January/2006 to April/2007 in the county of Campo Alegre, SC.

Tabela 1. Número médio de adultos de $H$. betulinus coletados nos tratamentos T I (testemunha), T II (catação), T III (duas aplicações anuais de $B$. bassiana), T IV (três aplicações anuais de $B$. bassiana), em um povoamento puro de erva-mate (I. paraguariensis), no município de Campo Alegre, SC.

Table 1. Mean number of H. betulinus adults detected in four areas of monoculture of yerba maté (I. paraguariensis) under the following treatments: T I (control), T II (hand picking), T III (two applications per year of B. bassiana), T IV (three applications per year of B. bassiana), in the county of Campo Alegre, SC.

\begin{tabular}{lcc}
\hline Tratamentos & Média \pm Erro padrão & Significância \\
\hline T I & $62,10 \pm 10,73$ & $\mathrm{a}^{*}$ \\
T II & $38,43 \pm 9,69$ & $\mathrm{~B}$ \\
T III & $28,62 \pm 7,31$ & $\mathrm{C}$ \\
T IV & $27,25 \pm 6,99$ & $\mathrm{C}$ \\
\hline * As médias seguidas pela mesma letra não diferem entre si pelo teste de Duncan a 5\% de probabilidade.
\end{tabular}

Gomm (2008), em testes com o mesmo formulado fúngico de B. bassiana (CG 716) em campo, observou que a dosagem de $50 \mathrm{ml}$ em $10 \mathrm{~L}$ de água, na concentração $10^{7}$ conídios $/ \mathrm{mL}$ e $0,5 \%$ de óleo, foi a mais eficaz, obtendo $33,26 \%$ de controle de $H$. betulinus com tempo médio de mortalidade de 34,05 dias.

Os resultados obtidos nesta pesquisa, corroborando o de outros autores, comprovam que o micoinseticida formulado com a linhagem CG 716 de B. bassiana apresenta um potencial elevado para o controle de $H$. betulinus quando aplicado no tronco e na base das erveiras, pelo menos duas vezes ao ano, em novembro e posteriormente em fevereiro. Além disso, como a catação manual é uma prática comum na cultura, com bons resultados, ela pode ser associada à aplicação do formulado fúngico, incrementando o controle da broca-da-erva-mate. 


\section{CONCLUSÕES}

- A utilização de B. bassiana na concentração de $10^{7}$ conídios $/ \mathrm{mL}$ representa uma medida eficaz para o manejo das populações de $H$. betulinus em áreas de cultivo intensivo de erva-mate.

- O cronograma de aplicação do formulado fúngico à base de B. bassiana a ser adotado deve incluir pelo menos duas aplicações anuais, nos meses de novembro e fevereiro.

- A catação manual representa uma medida que pode ser associada ao tratamento fúngico para o controle populacional da broca-da-erva-mate.

\section{AGRADECIMENTOS}

Aos pesquisadores Dra. Susete R. C. Penteado e Dr. Wilson Reis Filho, do Laboratório de Entomologia da Embrapa Florestas, pelas valiosas sugestões. Aos professores Dr. Juarez Gabardo e Dr. Júlio Valaski, pelas análises estatísticas. Ao Sr. Paulo Corrêa, pela concessão da área experimental. À Empresa Modo Battistella, pela disponibilização dos dados meteorológicos. À Indústria Turfal, pelo apoio financeiro, e à Dra. Maria Silvia P. Leite, pela produção do formulado fúngico. Ao CNPq, pela bolsa de doutorado concedida à primeira autora e de pesquisa para a segunda.

\section{REFERÊNCIAS}

ALVES, S. B. Controle microbiano de insetos. Piracicaba, SP: FEALQ, 1998. 1163 p.

BELLOWS, T. S. Restoring population balance through natural enemy introductions. Biological Control, v. 21, p. 199 - 205, 2001.

BORGES, L. R.; LÁZZARI, S. M. N.; LÁZZARI, F. A. Comparação dos sistemas de cultivo nativo e adensado de erva-mate, Ilex paraguariensis St.-Hil., quanto à ocorrência e flutuação populacional de insetos. Revista Brasileira de Entomologia, v. 47, p. 483 - 662, 2003.

CARPANEZZI, A. A. Cultura do mate no Brasil: conflitos e lacunas. In: H. WINGE, A. G.; FERREIRA, J. E. A.; MARIATH, E L. C.; TARASCONI (ed.). Erva-mate: biologia e cultura no Cone Sul. Porto Alegre, Ed. Universidade, p. 43 - 47, 1995.

DALlA SANTA, H. S.; SOUSA, N. J.; PITTNER, E.; DALlA SANTA, O. R.; SOCCOL, C. R. Controle biológico em pragas de Ilex paraguariensis (A. St.-Hil.) com fungo Beauveria sp. Revista Floresta, v. 39, p. 67 - 76, 2009.

DIAZ, C. I. F. Perspectivas del manejo integrado de plagas em yerba mate. In: CONGRESSO SULAMERICANO DA ERVA-MATE E II REUNIÃO TÉCNICA DO CONE SUL SOBRE A CULTURA DA ERVA-MATE, 1, 2, 1997. Anais... Curitiba, 1997, p. 371 - 390.

GALLO, D.; NAKANO, O.; SILVEIRA NETO, S.; CARVALHO, R. P. L.; BAPTISTA, G. C. V.; BERTI FILHO, E.; PARRA, J. R. P.; ZUCCHI, R. A.; ALVES, S. B.; VENDRAMIM, J. D.; LOPES, J. R. S.; OMOTO, C. Entomologia Agrícola. 2. ed. São Paulo: Agronômica Ceres, 2002. 649 p.

GOMM, P. C. Eficácia de diferentes dosagens do formulado fúngico à base de Beauveria bassiana (Vuill, 1912) no controle de adultos de Hedypathes betulinus (Klug, 1825) (Coleoptera: Cerambycidae). 32 f. Monografia - Universidade Estadual de Ponta Grossa, 2008.

HEADRICK, D. H.; GROEDEN, R. D. Biological control as a tool for ecosystem management. Biological Control, v. 21, p. 249 - 257, 2001.

IEDE, E. T. Considerações sobre a entomofauna da erva-mate (Ilex paraguariensis St.-Hil.) In: SEMINÁRIO SOBRE ATUALIDADES E PERSPECTIVAS FLORESTAIS - SILVICULTURA DA ERVA-MATE (Ilex paraguariensis ST.-HIL.), 1985, Colombo. Anais... Colombo: EMBRAPA/CNPF, 1985. $146 \mathrm{p}$. 
IEDE, E. T.; SOARES, C. M. S.; GRIGOLETTI JR., A.; AUER, C. G. Manual de identificação de pragas e doenças da erva-mate (Ilex paraguariensis St.-Hil.). Embrapa/CNPF. Colombo. 24 p. 2000.

LANZA, L. M.; MONTEIRO, A. C.; MALHEIROS, E. B. População de Metarhizium anisopliae em diferentes tipos e graus de compactação do solo. Ciência Rural, v. 34, p. 1757 - 1762, 2004.

LEITE, M. S. P.; SOARES, C. M. S.; IEDE, E. T.; PENTEADO, S. R. C.; CASTELlANO, C. Seleção de linhagens de fungos entomopatogênicos para o controle de Hedypathes betulinus (Klug, 1825) (Coleoptera: Cerambycidae) em laboratório e eficiência da linhagem selecionada em campo. In: CONGRESSO SUL-AMERICANO DA ERVA-MATE E REUNIÃO TÉCNICA DA ERVA-MATE. Encantado, 2, 3, 2000, RS. Anais... Encantado, 2000. p. 314 - 317.

LEITE, M. S. P.; PENTEADO, S. R. C.; OLIVEIRA, S. Avaliação de duas espécies de fungos entomopatogênicos para o controle de Hedypathes betulinus (Klug, 1825) (Coleoptera: Cerambycidae) em laboratório. In: Congresso SUL-AMERICANO DA ERVA-MATE. Chapecó, SC, 3,2003, Ed. dos Organizadores. Anais... Chapecó, 2003a. p. 16 - 19.

LEITE, M. S. P.; PENTEADO, S. R. C.; OLIVEIRA, S. Eficiência do fungo Beauveria bassiana (Bals.) Vuill. no controle de Hedypathes betulinus (Klug, 1825) (Coleoptera: Cerambycidae) em campo. CONGRESSO SUL-AMERICANO DA ERVA-MATE. 3, 2003, Chapecó, SC, Ed. dos Organizadores. Anais... Chapecó, 2003b.

LEITE, M. S. P.; IEDE, E. T.; PENTEADO, S. R. C.; ZALESKI, S. R. M.; CAMARGO, J. M. M.; RIBEIRO, D. R. Eficiência de Beauveria bassiana (Bals.) Vuill. formulado em óleo no controle de Hedypathes betulinus (Klug, 1825) (Coleoptera: Cerambycidae) em campo. 2006a. In: CONGRESO SUDAMERICANO DE LA YERBA MATE. 4, 2006, Anais... Missiones, 2006a. p. 269 - 272.

LEITE, M. S. P.; IEDE, E. T.; PENTEADO, S. R. C. Manejo integrado de pragas em erva-mate para o controle da broca-da-erva-mate, com ênfase ao uso de entomopatógenos. 2006b. In: CONGRESO SUDAMERICANO DE LA YERBA MATE. 4, 2006, Anais... Missiones, 2006b. CD-ROM.

OLIVEIRA, R. C.; ALVES, L. F. A.; NEVES, P. M. O. J. Suscetibilidade de Oligonychus yothersi (Acari: Tetranychidae) ao fungo Beauveria bassiana. Scientia Agricola, v. 59, p. 187 - 189, 2002.

RIBEIRO, M. M.; SANTOS, H. R.; DIODATO, M. A. Patogenicidade do fungo entomopatogênico Beauveria bassiana (Bals.). Vuill., em Hedypathes betulinus (Klug, 1825), praga da erva-mate, Ilex paraguariensis St.-Hill. Revista do Setor de Ciências Agrárias, v. 13, p. 229 - 231, 1994.

PENTEADO, S. R. C. Principais pragas da erva-mate e medidas alternativas para o seu controle. In: WINGE, H.; FERREIRA, A. G.; MARIATH, J. E. A.; TARASCONI, E. L. C. (ed.). Erva-mate: biologia e cultura no Cone Sul. Porto Alegre, Ed. Universidade, 356 p. 1995.

PENTEADO, S. R. C.; IEDE, E. T.; LEITE, M. S. P. Pragas da erva-mate: perspectivas de controle. In: CONGRESSO SUL-AMERICANO DA ERVA-MATE E REUNIÃO TÉCNICA DA ERVA-MATE. 2, 3, 2000, Encantado. Ed. dos Organizadores. Anais... Encantado, 2000. p. 27 - 38.

SOARES, C. M. S. Flutuação populacional, aspectos comportamentais e levantamento de inimigos naturais de Hedypathes betulinus (Klug, 1825) (Coleoptera: Cerambycidae) em um povoamento puro de erva-mate (Ilex paraguariensis St.-Hil.). 73 p. Tese (Doutorado) - Universidade Federal do Paraná, Curitiba, 1998.

SOARES, C. M. S.; IEDE, E. T. Perspectivas para o controle da broca-da-erva-mate Hedypathes betulinus (Klug, 1825) (Coleoptera: Cerambycidae). In: CONGRESSO SUL-AMERICANO DA ERVAMATE E REUNIÃO TÉCNICA DO CONE SUL SOBRE A CULTURA DA ERVA-MATE. 1, 2, 1997, Curitiba, Anais... Curitiba, 1997. p. 391 - 400.

SOARES, C. M. S.; IEDE, E. T.; SANTOS, H. R. Ocorrência natural dos fungos entomopatogênicos Beauveria bassiana e Metarhizium anisopliae sobre Hedypathes betulinus (Coleoptera: Cerambycidae). In: SICONBIOL - SIMPÓSIO DE CONTROLE BIOLÓGICO. 5, 1995, Foz do Iguaçu. Anais... Foz do Iguaçu, Embrapa. 1995. p. 81. 
FLORESTA, Curitiba, PR, v. 41, n. 2, p. 313-320, abr./jun. 2011.

Borges, L. R.; Lazzari, S. M. N.; Borges-Arriagada, L. R.; Iede, E. T. 